\title{
Recursos para avaliação da aprendizagem: estudo comparativo entre ambientes virtuais de aprendizagem
}

\author{
Gilmara Oliveira Maquiné ${ }^{1}$ \\ ${ }^{1}$ Instituto Federal de Educação, Ciência e Tecnologia do Amazonas \\ Programa de Pós Graduação em Ensino Tecnológico \\ gilmaramaquine@gmail.com
}

\begin{abstract}
This article presents a comparative study realized in Learning Management Systems (LMS) identified in the literature review. The purpose of this work is to verify which resources or instruments are used in these LMS to evaluate student learning. The research was conducted, in a qualitative perspective, by analyzing the data published in scientific articles, accessing the LMS, as well as collecting information from the Censo EAD.BR 2016. In the end, the existence of the use of evaluation through the attribution of notes, such as questionnaires, forums, tasks and etc. and also different instruments that enable more detailed monitoring of student learning, such as: checklist, rubrics, etc.
\end{abstract}

Resumo. Este artigo apresenta um estudo comparativo realizado em Ambientes Virtuais de Aprendizagem (AVAs) identificados na revisão da literatura. $O$ objetivo deste trabalho é verificar quais recursos ou instrumentos são utilizados nestes AVAs para avaliação da aprendizagem dos estudantes. A investigação foi conduzida, em uma abordagem qualitativa, por meio da análise dos dados publicados em artigos científicos, acessando os ambientes, bem como coleta de informações do Censo EAD.BR 2016. Ao final, identifica-se a existência do emprego de instrumentos de avaliação através da atribuição de notas, tais como questionários, fóruns, tarefas e etc. $e$ ainda instrumentos diferenciados que possibilitam o acompanhamento mais detalhado da aprendizagem dos estudantes, tais como: lista de verificação, rubricas e etc.

\section{Considerações iniciais}

A avaliação é considerada como parte integrante do processo ensino-aprendizagem, esses elementos ensino, aprendizagem e avaliação estão estreitamente relacionados, ao passo que qualquer alteração na avaliação reverbera nos outros (MORENO OLIVOS, 2016). Desta maneira, atualmente, para avaliar a aprendizagem faz-se necessário empregar diferentes métodos e instrumentos, considerando que este processo não se resume a um momento final.

Nesse aspecto, existe uma tendência em avaliar o aluno de forma integral identificada nas práticas de alguns países, tais como: Espanha e Estados Unidos. Porém, com a publicação da Base Nacional Comum Curricular (BNCC), em dezembro de 2017, a necessidade de por em prática esta tendência no cenário brasileiro vem se tornando um fator necessário a discussão, pois o teor do documento evidencia a abordagem de 
competências gerais e específicas que devem ser desenvolvidas nos estudantes ao longo da Educação Básica (BRASIL, 2017). Nesse sentindo, o fortalecimento na verificação ou adequações necessárias nos instrumentos de avaliação deve ser pautado considerando essas novas diretrizes.

Os Ambientes Virtuais de Aprendizagem (AVAs) ou Learning Management Systems (LMS) são softwares utilizados amplamente para popularização da modalidade de Educação a Distância, muito embora sua utilização possa ser vista em outras modalidades de ensino. Podem ser pagos ou gratuitos ou ainda criados para atender especificamente a demanda de uma instituição, porém, vale ressaltar que é caracterizado pelo ensino na internet. Nestes, são incluídas várias ferramentas/recursos para a avaliação sistemática do aprendizado dos alunos.

$\mathrm{Na}$ literatura existem diversos métodos para avaliar a utilização dos AVA, porém estes são focados em aspectos ergonômicos e de usabilidade desses ambientes. No estudo realizado por Lima, Merino e Triska (2018) os métodos de avaliação que têm tido maior incidência são: questionários, inspeções por guidelines e avaliação heurística. Porém, esse tipo de avaliação não faz referência ao teor pedagógico do processo avaliativo que constituí o ensino-aprendizagem, ou seja, tem sua aplicação a outras áreas de estudo que não fazem relação com este trabalho.

Este artigo tem como principal objetivo identificar quais recursos ou instrumentos são empregados nos AVAs no processo de avaliação da aprendizagem dos estudantes. A realização deste estudo foi viabilizada através de um estudo comparativo entre AVAs, identificados na literatura, com foco nos aspectos que envolvem a avaliação da aprendizagem dos alunos. Nesse sentido, a pergunta que norteia este estudo é: Quais recursos ou instrumentos são utilizados na avaliação do aprendizado de alunos em Ambientes Virtuais de Aprendizagem?

Para atender a finalidade do estudo, o artigo está organizado em três seções: a primeira apresenta diferentes definições de AVA e conceituações do processo avaliativo identificados na literatura; a segunda detalha a metodologia utilizada no estudo comparativo assim como apresenta a descrição dos AVA elencados; a terceira reúne os resultados identificados no estudo comparativo e discussões acerca das ferramentas e recursos para avaliação em AVA.

\section{Ambientes Virtuais de Aprendizagem}

O processo ensino-aprendizagem, atualmente, vem sendo realizado em locais além de uma sala de aula física, fazendo uso de Tecnologias da Informação e Comunicação (TIC), tais como os AVAs. Esses ambientes são descritos, de maneira geral, como sistemas que gerenciam alunos, professores e cursos. Nesse sentido, Junior e Marquesi (2009, p. 358) afirmam que os "ambientes virtuais de aprendizagem (AVA) podem ser definidos, na perspectiva do usuário, como ambientes que simulam os ambientes presenciais de aprendizagem com o uso das TICs. (...)”.

Os AVAs proporcionam aos alunos a aquisição de conhecimentos e habilidades passando por uma experiência de interação com um ambiente e as tecnologias nele contidas, na qual a comunicação ocorre sem a necessidade de que os dois pontos 
estejam conectados ao mesmo tempo (BARBOZA; SALES FILHO; SILVA, 2016, p. 96).

Esses ambientes são solidificados com o uso da internet como meio de disseminação de conhecimento e, segundo Gabardo, Quevedo e Ulbricht (2010) foram criados com o objetivo ampliar o alcance da educação e a possibilidade de chegar a localidades distantes.

Para Franco (2009, p. 48) AVA pode ser descrito como uma plataforma de ensino a distância e acrescenta alguns acrônimos a essa definição, tais como "plataforma de e-learning, SGC ou LMS é um software que oferece um conjunto de ferramentas com as quais o professor e/ou designer poderá criar um curso completamente on-line ou um componente on-line complementar a um curso presencial"'.

$\mathrm{Na}$ perspectiva do ensino-aprendizagem, a principal função dos AVA é proporcionar o acesso aos conteúdos utilizados na realização das aulas compartilhando com os alunos por meio de "recursos e ferramentas que podem variar de acordo com as necessidades dos cursos oferecidos. Eles podem atender tanto aos sistemas educacionais a distância quanto ao presencial como suporte as atividades de sala de aula" (LIMA; MERINO; TRISKA, 2018, p.135).

Nesse cenário, Pavezi et al (2011) resumem as principais ferramentas que constituem os AVA: chats, vídeo-aulas, fórum, lista de discussão, correio eletrônico, mural, enquete, portfólio, perfil e FAQ (Frequently Asked Questions).

Segundo informações do Censo 2016, publicado pela Associação Brasileira de Educação a Distância, o AVA "é um recurso fundamental tanto para a distribuição de conteúdos quanto para a interação com os alunos". Ainda neste relatório são apresentados dados referentes aos tipos de AVA, na categoria administrativa, identificados nas instituições de ensino detalhando os tipos de ambientes:

i) ambiente de aprendizagem aberto (software livre), customizado pela própria instituição; ii) ambiente de aprendizagem proprietário; iii) ambiente de aprendizagem aberto (software livre), customizado por terceiros; iv) ambiente de aprendizagem criado pela própria instituição; v) ambiente de aprendizagem aberto (software livre), sem customização (CENSO, 2016, p.121).

Dentre os tipos elencados, a maioria das instituições reunidas no estudo supramencionado, cerca de 60, utiliza ambientes de aprendizagem abertos (software livre), na qual a customização é realizada pela própria instituição. Diante do exposto, na próxima subseção vamos detalhar modalidades de avaliação que são utilizadas em AVAs.

\subsection{Avaliação da Aprendizagem em AVAs}

$\mathrm{Na}$ conjuntura apresentada faz-se necessário evidenciar e elencar algumas conceituações acerca de avaliação de aprendizagem no âmbito educacional. Nesse sentido, Roque (2004) afirma existirem vários modelos de avaliação, criados na história educacional, baseando-se sempre em três paradigmas: "positivismo ou quantitativo, interpretativo ou qualitativo, e a teoria crítica". 
No Brasil a avaliação para a Educação Básica é norteada pelo Art. 24 da Lei no 9.394, de 20 de dezembro de 1996 - LDB (BRASIL, 1996), qual descreve que a avaliação deve ser "contínua e cumulativa do desempenho do aluno, com prevalência dos aspectos qualitativos". A complementação desse sentido de avaliação proposto pela LDB, trecho supramencionado, se dá com o Art. 35 da Lei no 13.415 de 16 de fevereiro de 2017, que acrescenta

$$
\begin{aligned}
& \S 8^{\circ} \text { Os conteúdos, as metodologias e as formas de avaliação processual e } \\
& \text { formativa serão organizados nas redes de ensino por meio de atividades } \\
& \text { teóricas e práticas, provas orais e escritas, seminários, projetos e atividades } \\
& \text { on-line, de tal forma que ao final do ensino médio o educando demonstre: } \\
& \text { I - domínio dos princípios científicos e tecnológicos que presidem a produção } \\
& \text { moderna; } \\
& \text { II - conhecimento das formas contemporâneas de linguagem (BRASIL, 2017). }
\end{aligned}
$$

Ferreira (2003, p. 27) afirma que "a avaliação do aluno sempre foi algo que desafiou e estimulou professores, na busca de algo que realmente perceba a real situação do aprendizado do aluno". Apresenta ainda a classificação proposta a partir da perspectiva da Taxonomia Bloom para as avaliações, sendo: diagnóstica, somativa e formativa. Cada uma dessas tem função, propósito e momento de realização. A diagnóstica tem a função de diagnosticar, com o proposito de identificar a "presença ou ausência de pré-requisitos para novas aprendizagens", e geralmente é realizada no inicio do ano ou semestre letivo. Já a somativa tem a função de classificar, com o propósito de "Classificar os resultados de aprendizagem alcançados pelos alunos, de acordo com níveis de aproveitamento estabelecidos", e são realizadas ao final do ano ou semestres letivos. A formativa tem a função de controlar, com o propósito de "Constatar se os objetivos estabelecidos foram alcançados pelos alunos. Fornecer dados para aperfeiçoar o processo ensino-aprendizagem", são realizadas durante o ano, ao longo do processo ensino-aprendizagem.

No que tange a avaliação em ambientes virtuais de aprendizagem, Pallof e Pratt (2015, p.100) afirmam que avaliar na sala de aula on-line utilizando os meios tradicionais, pode não ser a melhor opção, consideram: "testes e quizzes podem não ser a melhor medida de aquisição de conhecimento e de aplicação on-line".

Segundo Behar e Bassani (2009, p. 100), o processo avaliativo realizado em AVAs, pode ser compreendido a partir de três aspectos: "a) avaliação por meio de testes online; b) avaliação da produção individual de estudantes; c) análise das interações entre alunos, a partir das mensagens postadas/trocadas por meio das diversas ferramentas de comunicação".

\section{Metodologia}

A investigação de caráter qualitativo foi conduzida em duas fases. A primeira se caracteriza por uma revisão da literatura a partir da análise de dados publicados em artigos científicos. A segunda se caracteriza pele seleção dos AVAs qual deu-se por amostragem intencional, conforme proposto por Gil (2002). O autor propõe uma amostra ideal para análise entre 4 e 10 objetos de pesquisa. Tomando este critério como parâmetro, dos 15 AVAs identificados na pesquisa serão detalhados sete (7) mais citados na amostra de artigos científicos e levando em consideração as categorias: i) ambiente de aprendizagem aberto (software livre), customizado pela própria instituição; 
e ii) ambiente de aprendizagem proprietário; elencadas no Censo EAD.BR 2016. Foram selecionados os seguintes: i) Amadeus, ii) Canvas, iii) E-Proinfo, iv) Google Classroom, v) Moodle, vi) Rooda e vii) Teleduc, qual passaremos a detalhar.

i) Amadeus: Descrito como um Sistema Gerenciador de Aprendizagem ou Learning Management System (LMS). Criado em 2007, pelo Grupo de Ciências Cognitivas e Tecnologia Educacional (CCTE), do Centro de Informática da Universidade Federal de Pernambuco. O projeto foi desenvolvido e distribuído sob a licença GNU/GPL e tem suporte multi-idioma.

ii) Canvas: Software desenvolvido pela empresa Instructure, lançado em 2011 no mercado de e-learning. Possui uma versão de acesso gratuito e outra qual é necessário investir em pagamento. Na primeira, para ter acesso é necessário apenas realizar o cadastro na plataforma disponível na internet. Na segunda, será realizado um orçamento com entre a instituição e a empresa. Segundo Mesquita, Junior e Gara (2014) a versão aberta é satisfatória para oferecer cursos online. Destacam os recursos de gestão, atividades, diferentes configurações de notificação. Apontam como diferencial a área de avaliação speedgrade, onde são reunidos todos os alunos e permite que o professor tenha uma visão ampla da turma.

iii) E-Proinfo: Ambiente colaborativo de aprendizagem criado pelo Laboratório de Tecnologia da Informação e Mídias Educacionais (Lab Time da Universidade Federal de Goiás (UFG), em parceria com o Ministério da Educação (MEC). Proporciona a colaboração das secretarias de educação estaduais, criam cursos voltados para formação de professores.

iv) Google Classroom: Ambiente criado pelo Google em 2014, que faz parte do conjunto de soluções GSuite (em algumas literaturas podemos encontrar a nomenclatura "Google For Education" porém esta foi substituída pela atual). O objetivo é proporcionar uma simplificação das tarefas, colaboração e promover a comunicação contínua para tornar o ensino mais produtivo e significativo.

v) Moodle (Modular Object-Oriented Dynamic Learning Environment): Moodle é um software livre que tem como objetivo apoiar o ensino. Criado em 2001, vem sendo utilizado por diversas instituições de ensino para disseminar a educação à distância. Atualmente, o software é utilizado em mais de 100 mil sites hospedados em mais de 200 países, segundo informações do site da organização mantenedora.

vi) Rooda (Rede Cooperativa de Aprendizagem): Ambiente de Ensino a distância da Universidade Federal do Rio Grande do Sul (UFRGS). Foi desenvolvido com o objetivo de fornecer um ambiente virtual e ferramentas aos professores e alunos da universidade. Em seu escopo o professor pode fazer as avaliações por atribuição de conceitos.

vii) TelEduc: Sistema com interface baseada para web, desenvolvido pelo Núcleo de Informática Aplicada à Educação do Instituto de Computação da Unicamp. $\mathrm{O}$ ambiente foi iniciado a partir da dissertação de mestrado (Alessandra de Dutra e Cerceau), em 1998 e proporcionou diversos frutos aos longos dos anos seguintes. 


\section{Resultados e Discussão}

Nesta seção, apresentamos os resultados do estudo comparativo entre os AVAs. O Quadro 1 reúne informações coletadas a partir da análise de cada AVA e evidencia os principais métodos de avaliação disponíveis nestes.

Quadro 1 - Quadro comparativo dos AVAs

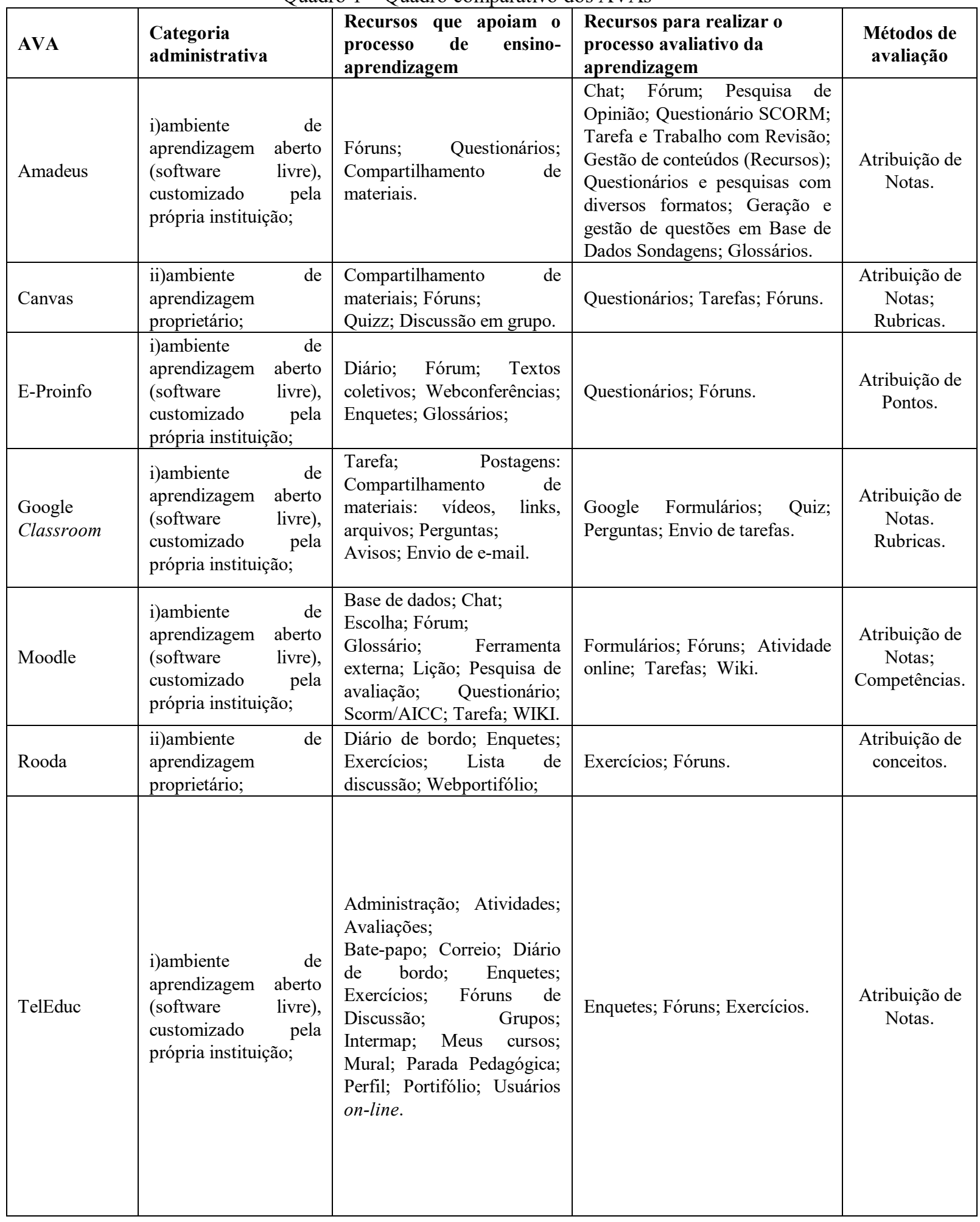


Após análise dos AVA evidenciamos algumas características de cada um desses, e destacaremos os seus respectivos diferenciais e pontos de atenção.

Os procedimentos de avaliação do AVA i) Amadeus, levam em consideração os pontos de vistas dos participantes e seus pares. São considerados diagnósticos realizados continuamente. Como diferenciais do Amadeus podemos afirmar que o idioma em português; a possibilidade do uso de mídias; Interface intuitiva; Destina-se tanto para o ensino presencial quanto para a distância, e em seu formato atual é recomendado o uso para o ensino híbrido. Outro aspecto importante é o fato desse AVA estar disponível no Portal do Software Público, o que aumenta o alcance e abrangência de público. No que concerne à interação no AVA Amadeus "A interação entre os usuários, e destes, com o conteúdo no ambiente permite a execução de novas estratégias de ensino e de aprendizagem orientadas por teorias construtivistas ou sócio interacionista do desenvolvimento humano" (MELO FILHO, et al. , 2014, p.111). Neste percurso podemos identificar alguns pontos de atenção, tais como: a necessidade de investimento em infraestrutura de TIC para instalação e configuração do software e a institucionalização do uso.

Com relação ao AVA ii) Canvas, uma das funcionalidades disponíveis à avaliação é a utilização de rubricas. Estas são previamente cadastradas pelos professores e no momento que o aluno acessa a atividade, antes de responder, tem a visualização da grade de avaliação. Além desse diferencial no processo de avaliação, evidenciamos como vantagem o acesso via internet, sem a necessidade de possuir uma infraestrutura física de TIC na instituição. Alguns pontos de atenção que podemos apontar é a necessidade de pagamento de licença para uso completo da ferramenta e o idioma padrão inglês, que pode ser visto como barreira para alguns usuários.

O iii) E-Proinfo, no que tange a avaliação o Manual do sistema apresenta uma descrição sobre as 'categorias de avaliação', onde o professor pode ser realizar a atribuição de notas aos alunos. Pode ser verificado que o sistema apresenta a opção para avaliação por atribuição de pontos. Principais diferenciais identificados: ambiente web colaborativo; ambiente para instituições de ensino em municípios e estados. Ponto de atenção que podemos apontar é que a plataforma foi criada para a colaboração entre profissionais da educação, porém para participar dos cursos a pessoa deve estar vinculada a unidade que esta oferecendo que pode vir a limitar o acesso ao ambiente.

Quanto ao AVA iv) Google Classroom, concordamos com as vantagens elencadas por Sousa Junior et al (2017) no seu estudo do Google Classroom e destacamos estas: Configuração simples e acesso restrito, Fluxo de atividades sem papel, Melhoria na organização, Feedback imediato, Fácil acesso e seguro. Para além dessas, destacamos também que em seu ambiente nativo, ou seja, da maneira que foi desenvolvida, o Google Classroom possibilita a avaliação de atividades apenas por notas. Porém, é possível fazer uso de complementos (plugins) que proporcionam a avaliação utilizando rubricas. Estes plugins estão em inglês e o seu uso é pouco difundido e divulgado em publicações científicas. Como exemplo desses citamos: Doctopus e Goobric. O Doctopus é um plugin gratuito para o Google Sheets que foi criado com o objetivo ajudar os professores a coletar, salvar e acompanhar as informações das atividades realizadas pelos estudantes no Google Classroom em uma 
planilha. Já o Goobric também é um plugin gratuito, que funciona em conjunto com o Doctopus, e possibilita a avaliação das atividades a partir da criação de rubricas.

O v) Moodle é um AVA de uso muito popular atualmente conforme descrito na descrição preliminar na sessão acima. Quanto aos recursos de avaliação da aprendizagem, majoritariamente o que podemos perceber o uso do Moodle é a avaliação por meio de atribuição de notas as atividades desenvolvidas pelos alunos.

Para Silva (2013, p.19) o Moodle "trabalha com uma perspectiva dinâmica da aprendizagem em que a pedagogia socioconstrutivista e as ações colaborativas ocupam lugar de destaque". O autor destaca que o Moodle é um software de fácil utilização, porém, a criação de cursos no ambiente é necessário planejamento e cuidados especiais referentes ao design educacional e estrutura das páginas HTML. Construído baseado no modelo social de aprendizagem, com foco central no aluno. Nesse AVA, os cursos podem ser organizados em semana ou tópico, e favorece o uso de ferramentas de discussão e compartilhamento de materiais (FRANCO, 2009). Porém, em 2015 a funcionalidade para avaliação por competências, no qual podemos cadastrar competências e associá-las às tarefas foi lançada como uma opção nativa do Moodle versão 3.1. A utilização desta funcionalidade ainda não ganhou o destaque necessário nas publicações.

Destacamos como diferenciais do Moodle a ampla utilização do ambiente, em diversos países e instituições, e a dispensa de pagamento de licença para uso. Como pontos de atenção, identificamos a obrigatoriedade de ter uma infraestrutura física para instalação e funcionamento do ambiente; a contratação de pessoal especializado tanto para área de tecnologia da informação quanto para área pedagógica; os recursos que apoiam o processo avaliativo disponíveis no ambiente são direcionados a atribuição de notas; e, por fim, a carência de estudos aprofundados dos recursos de avaliação por competências.

O ambiente vi) Rooda apresenta uma metodologia de avaliação por conceitos, no qual o professor utiliza as funcionalidades 'Anotações' e 'Sistema Automático'. Na primeira o professor deve escolher o tipo de codificação que será utilizada na avaliação. $\mathrm{Na}$ segunda as notas são codificadas utilizando o sistema numérico. Uma vantagem que podemos destacar neste AVA é que ele foi construído voltado para realidade da instituição e por este motivo o suporte é facilitado. Quanto à desvantagem, neste estudo, apontamos a questão de ser necessária a utilização de plugins para acesso ao ambiente.

No AVA vii) Teleduc, a ferramenta 'Atividades' é descrita como o elemento central do ambiente. Nesse sentido esta pode ser descrita como um módulo do Teleduc que reúne todas as atividades realizadas pelos participantes. Já o módulo 'Avaliações', são realizadas as avaliações das atividades realizadas. Este módulo foi criado pensando em avaliações formativas, baseando-se em atribuição de notas. Identifica-se com diferenciais desse ambiente a criação realizada no Brasil e manutenibilidade por uma universidade. Existe vasto material acadêmico e de suporte técnico da ferramenta. $\mathrm{O}$ uso gratuito é outra vantagem. Mesquita, Junior e Gara (2014, p. 44), consideram o Teleduc "um ambiente enxuto, mas extremamente flexível". Os autores afirmam ainda que a estrutura do ambiente possibilita a "a implementação de diferentes cenários de aprendizagem (instrucional, construtivista e interacionista)" (MESQUITA; JUNIOR; GARA, 2014, p. 44). 
IX Congresso Brasileiro de Informática na Educação (CBIE 2020)

Anais do XXVI Workshop de Informática na Escola (WIE 2020)

\section{Considerações finais}

Diante do exposto, foi possível conceituar AVAs e explorar as características dos elencados no estudo. Identificou-se que a maioria dos AVAs analisados disponibilizam em seu escopo os métodos de avaliação por atribuição de notas, tais como questionários, formulários automáticos e etc. De maneira geral, a modalidade ou tipo de avaliação não ficou claro nos AVAs, ou seja, o processo avaliativo empregado nesses ambientes decorre da escolha das funcionalidades que apoiam o processo ensinoaprendizagem. Essa escolha está alinhada as necessidades de ensino-aprendizagem complementando o planejamento e não necessariamente esta diretamente relacionada às funcionalidades ou recursos de avaliação. Outro aspecto identificado nos AVAs: Canvas, Google Classroom, Moodle e Rooda, durante o estudo, foi a utilização de outros métodos de avaliação: avaliação por rubricas, competências e conceitos e estes destacam-se como diferenciais destas plataformas. Vale ressaltar ainda a necessidade de identificar como as instituições estão fazendo uso desses métodos diferenciados, pois com a publicação da Base Nacional Comum Curricular (BNCC) no Brasil deveremos enfrentar o desafio de avaliar para além das notas e com isso os AVAs necessitam adequar-se para atender a essa demanda das escolas.

\section{Referências}

BARBOZA, E. J. S. and SALES FILHO, A. G. and SILVA, M. da P. (2016) Comparação entre os principais AVA's quanto a interatividade. In: International Conference on Engineering and Technology Education, 14., Salvador. Anais... Salvador: UNEB, 2016. p. 96-100. Disponível em: $<$ https://bit.ly/2WYdf5T>. Acesso em: 06 jun. 2019.

BEHAR, P. A. and BASSANI, P. (2009) Avaliação da aprendizagem em ambientes virtuais. In: BEHAR, P. et al. Modelos pedagógicos em educação a distância. Porto Alegre: Artmed, p. 93-113.

BRASIL. (2017) Base Nacional Comum Curricular. Brasília: MEC. Disponível em: $<$ https://bit.ly/1SRlt6L $>$. Acesso em: 25 mar. 2019.

BRASIL. (1996) Lei $n^{\circ} 9.394$ de 20 de dezembro de 1996. Brasília: MEC. Disponível em: <https://bit.ly/2OQNkct>. Acesso em: 17 fev. 2019.

BRASIL. (2017) Lei $n^{o} 13.415$ de 16 de fevereiro de 2017. Brasília: MEC, 2017. Disponível em: $<$ https://bit.ly/2Ldmr3T $>$. Acesso em: 17 fev. 2019.

CENSO, E. A. D. BR: Relatório Analítico da Aprendizagem a Distância no Brasil 2016 (2016). Censo EAD. BR: Analytic Report of Distance Learning in Brazil. Curitiba: Ibpex. Disponível em:<https://bit.ly/2LDkWK5> Acesso em: 15 mar. 2016.

LIMA, G. F. de A. and MERINO, E. A. D. and TRISKA, R. (2018) Métodos mais usados para avaliações de Ambientes Virtuais de Aprendizagem (AVAs). Human Factors in Design, v. 7, n. 13, p. 132-147. Disponível em: $<$ https://bit.ly/31U5vW0>. Acesso em: 06 jun. 2019.

FERREIRA, T. B. (2003) Gerenciador de Avaliações: Uma Ferramenta de Auxílio à Avaliação Formativa para o Ambiente de Educação a Distância TelEduc. Campinas: 
UNICAMP, 129 f. Dissertação de Mestrado em Computação. Disponível em: $<$ https://bit.ly/2Xzz1B6> Acesso em: 30 maio 2019.

FRANCO, C. de P. (2009) O uso de um ambiente virtual de aprendizagem no ensino de inglês: além dos limites da sala de aula presencial. Dissertação de Mestrado, Programa Interdisciplinar de Pós-Graduação em Linguística Aplicada, Faculdade de Letras, Universidade Federal do Rio de Janeiro. Rio de Janeiro. Disponível em: $<$ https://bit.ly/2IRf0Op $>$. Acesso em: 25 maio 2019.

GABARDO, P. and QUEVEDO, S. R. P. de and ULBRICHT, V. R. (2010) Estudo Comparativo das Plataformas de Ensino-Aprendizagem, Enc. Bibli: R. Eletr. Bibliotecon. Ci. Inf., v.1, n.esp, p.65-84.

GIL, A. C. (2002) Métodos e técnicas de pesquisa social. São Paulo: Editora Atlas.

JUNIOR, C. F. de A. and MARQUESI, S. C. (2009) Atividades em ambientes virtuais de aprendizagem: parâmetros de qualidade. In: LITTO, F. M.; FORMIGA, M. (Org). Educação a Distância: o estado da arte. São Paulo: Pearson Education do Brasil, p. 358-368.

MElO FILHO, I., CARVAlHO, R., GOMES, A., Perris, P., RODRIGUES, R., \& FELICIANO, F. (2014). Análise comparativa da Usabilidade dos Ambientes de Gestão da Aprendizagem Amadeus e Moodle. Revista Brasileira de Informática na Educação, 22(01), 107. doi:http://dx.doi.org/10.5753/rbie.2014.22.01.107. Acesso em: 23 set. 2020.

MESQUITA, D. and JUNIOR, D. P. and GARA, E. B. M. (2014) Ambiente Virtual de Aprendizagem: conceitos, normas, procedimentos e práticas pedagógicas no Ensino a Distância, 1 ed., São Paulo: Érica.

MORENO OLIVOS, T. (2016) Evaluación del aprendizaje y para el aprendizaje: reinventar la evaluación en el aula. México: UAM, Unidad Cuajimalpa.

PALLOFF, R. M. and PRATT, K. (2015) Lições da sala de aula virtual: as realidades do ensino on-line. Porto Alegre: Penso.

PAVEZI, A. M. et al. (2011) O uso das ferramentas do Ambiente Virtual de Aprendizagem pelos acadêmicos dos cursos de administração e processos gerenciais do NEAD-CESUMAR. In: Congresso Nacional de Educação a Distância, 17. Manaus. Disponível em: <https://bit.ly/2X1Yy6M>. Acesso em: 04 jun. 2019.

ROQUE, G. O. B. (2004) Uma proposta de um modelo de avaliação de aprendizagem por competências para cursos a distância baseados na web. Rio de Janeiro:UFRJ, 156 f. Dissertação de Mestrado em Informática. Disponível em: $<$ https://bit.ly/2N9pywm>. Acesso em: 3 abr. 2020.

SILVA, R. S. (2013) Moodle para autores e tutores: educação a distância na web 2.0. São Paulo: NOVATEC.

SOUSA JUNIOR, A. et al. (2017) Google Suite for education: trazendo o Google Classroom como uma perspectiva para as salas de aula usando os dispositivos móveis. In: Congresso sobre Tecnologias na Educação, 2., Mamanguape. Anais... Mamanguape: Universidade Federal da Paraíba - Campus IV, 2017, p.125-137. Disponível em: <https://bit.ly/2xlhz4V>. Acesso em: 04 jun. 2019. 